\title{
State-Dependent Impulsive Control Strategies for a Tumor-Immune Model
}

\author{
Kwang Su Kim, ${ }^{1}$ Giphil Cho, ${ }^{1}$ Lin-Fei Nie, ${ }^{2}$ and Il Hyo Jung ${ }^{1}$ \\ ${ }^{1}$ Department of Mathematics, Pusan National University, Busan 609-735, Republic of Korea \\ ${ }^{2}$ College of Mathematics and Systems Science, Xinjiang University, Urumqi 830046, China
}

Correspondence should be addressed to Il Hyo Jung; ilhjung@pusan.ac.kr

Received 23 May 2016; Accepted 1 September 2016

Academic Editor: Ryusuke Kon

Copyright (C) 2016 Kwang Su Kim et al. This is an open access article distributed under the Creative Commons Attribution License, which permits unrestricted use, distribution, and reproduction in any medium, provided the original work is properly cited.

Controlling the number of tumor cells leads us to expect more efficient strategies for treatment of tumor. Towards this goal, a tumorimmune model with state-dependent impulsive treatments is established. This model may give an efficient treatment schedule to control tumor's abnormal growth. By using the Poincaré map and analogue of Poincaré criterion, some conditions for the existence and stability of a positive order-1 periodic solution of this model are obtained. Moreover, we carry out numerical simulations to illustrate the feasibility of our main results and compare fixed-time impulsive treatment effects with state-dependent impulsive treatment effects. The results of our simulations say that, in determining optimal treatment timing, the model with state-dependent impulsive control is more efficient than that with fixed-time impulsive control.

\section{Introduction}

Cancer is a class of diseases characterized by out of control cell growth. Abnormal cells divide without control and are able to invade other tissues. It can also spread to other parts of the body through the blood and lymph systems. Cancer is a leading cause of death worldwide. In the past decades, doctors are trying to cure cancer but it has been difficult. Among many treatments for cancer, immunotherapy is a treatment method by using immune system in a body to fight cancer cells. Immune system recognizes cancer cells and leads to destruction of cancer cells before cancer cells are big enough to see. However, since the mechanism of immune system in the body is very complex, it is difficult to find an efficient treatment schedule to eradicate some cancers. So, in order to find such schedule, a mathematical model describing dynamics of tumor-immune interactions would provide a new strategy for treatment of cancer.

In the past, mathematical modeling about tumorimmune interactions has been studied by many scholars (see [1-3]). Particularly, Gałach [4] provided three kinds of different models which describe a competition between the tumor and immune cells by using a mathematical model with delay. Actually they investigated the stability of the steady state and observed a state of the "returning" tumor in the model with time delay. In several papers, there are several mathematical models that describe treatment effects: immunotherapy, chemotherapy, radiation therapy, and so on $[1,5]$.

In real world, such treatments need not once but periodic schedules. In particular, the impulsive differential equations can describe well such periodic treatments and pulse vaccination. Disease mathematical models using time impulsive differential equations have been studied by many researchers. For example, Meng and Chen [6] suggested a delayed SIR epidemic model with impulsive effect and analyzed the dynamic behaviors of the model. For SI model, Pei et al. [7] analyzed the dynamic behaviors by an impulsive delayed SI model. In Gao et al. [8], they proposed the periodic therapy from antiretroviral drugs for HIV by using impulsive differential equations. Qiao et al. [9] suggested a Hepatitis $B$ virus infection model with impulsive vaccination and time delay. Also they showed the existence of a periodic solution of the model and derived sufficient conditions that Hepatitis B virus will be eliminated or persistent. Huang et al. [10] suggested two novel mathematical models by using an impulsive control term for injection of insulin and showed the existence and stability of the periodic solution of the model. 
A noticed fact is that the treatment of different cancers has different ways. For some cancers that cannot be cured completely, a natural idea is therefore to keep the density of tumor cells at a low level to slow the progression of cancers. Based on this idea, Tang et al. [11] proposed an SIR epidemic model with state-dependent pulse control strategies and demonstrated that the combination of pulse vaccination and treatment is optimal in terms of cost under certain conditions, and the existence and stability of periodic solution with the maximum value of the infective no greater than threshold value RL are studied. Nie et al. [12] proposed an SIR epidemic model with state-dependent pulse vaccination and proved the existence and the stability of a positive periodic solution for the model. Additionally, in tumor treating, people have high death rate if they have tumor cells too much. So it is important that we control to reduce the number of tumor cells below the value of hazardous. Therefore, to describe tumorimmune systems with treatments, state-dependent impulsive differential equation is more appropriate than fixed-time dependent impulsive differential equation. However, the mathematical models for tumor-immune interactions with state-dependent impulsive treatments are rare. Additionally, state-dependent impulsive control strategies are also used in the treatment and control of viral diseases. For example, Nie et al. [13] proposed two novel virus dynamics models with state-dependent control strategies and analytically proved the existence and orbit stability of semitrivial periodic solution and positive periodic solution. Theoretical results showed that the density of infected cells or free virus can be kept within a low level over a long period of time by adjusting control strength. Tang et al. [14] constructed a mathematical model with state-dependent impulsive interventions where the comprehensive therapy involving combining surgery with immunotherapy is considered. In it, authors examined the global dynamics of the immune tumour system with statedependent feedback control, including the existence and stability of the semitrivial order-1 periodic solution and the positive order- $k$ periodic solution.

Inspired by the above discussion, the objective of this study is to develop a tumor-immune model with statedependent impulsive treatment: immunotherapy and chemotherapy by using state-dependent impulsive differential equations, find some conditions for the existence and stability of periodic solution, and compare between state-dependent impulsive control and fixed-time impulsive control for control of tumor.

The rest of this paper is organized as follows: In Section 2, we give a mathematical model for the tumor-immune system with state-dependent impulsive control strategies, which is based on the Gałach's model [4], and describe the parameters used in this model. In Section 3, for the main results, we show the existence and stability of a positive order-1 periodic solution. In Section 4, we carry out some numerical simulations to illustrate the main results and compare a fixedtime pulse treatment with a state-dependent pulse treatment. Finally, the conclusion is given in Section 5.

\section{Mathematical Model of Tumor-Immune Dynamics}

Gałach's autonomous tumor-immune dynamics model is given by the following differential equations [4]:

$$
\begin{aligned}
& \frac{\mathrm{d} E(t)}{\mathrm{d} t}=\sigma+\omega T(t) E(t)-\delta E(t) \\
& \frac{\mathrm{d} T(t)}{\mathrm{d} t}=\alpha T(t)[1-\beta T(t)]-T(t) E(t) .
\end{aligned}
$$

Gałach changed the model proposed in [3] by replacing the Michaelis-Menten form of function with a Lotka-Volterra form. Here $E(t), T(t)$ represent the numbers of immune cells and tumor cells, respectively, and parameter $\sigma>0$ denotes the rate of immune cell proliferation, parameter $\omega>0$ describes the immune response to the appearance of the tumor cells, parameter $\delta>0$ is a rate of immune cell turnover, parameter $\alpha>0$ is a growth rate of tumor cells, and parameter $\beta>0$ denotes the inverse of tumor cell's carrying capacity; $1 / \beta$ is the mean tumor cell's carrying capacity.

On the stability of an equilibrium, Gałach [4] has the following results.

Lemma 1. (i) If $\omega>0$ and $\alpha \delta<\sigma$, then model (1) has a trivial equilibrium $P_{0}(\sigma / \delta, 0)$ which is stable.

(ii) If $\omega>0$ and $\alpha \delta>\sigma$, then model (1) has an unstable equilibrium $P_{0}$ and a stable positive equilibrium $P_{1}\left(E_{1}, T_{1}\right)$, where

$$
\begin{aligned}
E_{1} & =\frac{\alpha(\beta \delta+\omega)-\sqrt{\Delta}}{2 \alpha \beta \omega}, \\
T_{1} & =\frac{-\alpha(\beta \delta-\omega)+\sqrt{\Delta}}{2 \omega}, \\
\Delta & =\alpha^{2}(\beta \delta-\omega)^{2}+4 \alpha \beta \sigma \omega>0 .
\end{aligned}
$$

(iii) If $\omega<0, \alpha \delta>\sigma$ and $\alpha(\beta \delta-\omega)^{2}+4 \beta \sigma \omega>0$, then model (1) has an unstable equilibrium $P_{0}$ and a stable positive equilibrium $P_{1}\left(E_{1}, T_{1}\right)$, where

$$
\begin{aligned}
E_{1} & =\frac{\alpha(\beta \delta+\omega)-\sqrt{\Delta}}{2 \alpha \beta \omega}, \\
T_{1} & =\frac{-\alpha(\beta \delta-\omega)+\sqrt{\Delta}}{2 \omega}, \\
\Delta & =\alpha^{2}(\beta \delta-\omega)^{2}+4 \alpha \beta \sigma \omega>0 .
\end{aligned}
$$

It is well known that immunotherapy and chemotherapy are effective to control the tumor cells. Chemotherapy attacks not only tumor cells but also normal cells and immunotherapy is applied by injection of immune cells. Therefore, cancer patients need periodic treatments for a long time. For the reality, a state-dependent impulsive control strategy for tumor abnormal growth is proposed here, rather than the usual continuous control or pulse fixed-time control strategy. The treatment strategy is based on the following assumption. 
(A) When the amount of tumor cell reaches the hazardous threshold value $H(>0)$ at time $t_{i}(H)$ at the $i$ th time, immunotherapy and chemotherapy are taken and the amounts of immune cells and tumor cells abruptly turn to $(1-p) E\left(t_{i}^{-}(H)\right)+\tau$ and $\left((1-q) T\left(t_{i}^{-}(H)\right)\right)$, respectively, where $p, q \in(0,1)$ are the chemotherapy intensity and $\tau \in(0,1)$ is the amounts of immunotherapy, respectively.

Under assumption (A), we build the control process that is modeled by the following differential equation with statedependent impulsive effects:

$$
\begin{array}{ll}
\frac{\mathrm{d} E(t)}{\mathrm{d} t}=\sigma+\omega T(t) E(t)-\delta E(t) & \\
\frac{\mathrm{d} T(t)}{\mathrm{d} t}=\alpha T(t)[1-\beta T(t)]-T(t) E(t) & T<H, \\
\Delta E(t)=E(t)-E\left(t^{-}\right)=-p E\left(t^{-}\right)+\tau & \\
\Delta T(t)=T(t)-T\left(t^{-}\right)=-q T\left(t^{-}\right) & T=H .
\end{array}
$$

Remark 2. We indicate that we do not know a priori time of pulse treatments of chemotherapy and immunotherapy since it is taken at the time when the number of tumor cells $T$ reaches the threshold value $H$. So the pulse treatments of chemotherapy and immunotherapy time obviously depend on the solution and our model adapts state-dependent pulse treatments of chemotherapy and immunotherapy.

Remark 3. The control parameters $p, q, \tau$ and hazardous threshold $H$ rely heavily on the characteristics of various cancer types. The choices of these values are very important, which are closely geared to the growth or elimination of cancer cells.

Lemma 4. Each component of solution $(E(t), T(t))$ of model (4) with nonnegative initial condition $\left(E_{0}, T_{0}\right)=\left(E\left(t_{0}\right), T\left(t_{0}\right)\right)$ is positive for every $t \geq 0$.

Proof. For any nonnegative initial condition $\left(E_{0}, T_{0}\right)$, suppose that $(E(t), T(t))$ is a solution of model (4) with the positive initial conditions $\left(E_{0}, T_{0}\right)$; then relation of solution $(E(t), T(t))$ with line $K: T=H$ is one of the following cases.

(i) The Solution $(E(t), T(t))$ Intersects Line $K$ Finite Times. Since $(E(t), T(t))$ intersects line $K$ finite times and the positive equilibrium $P_{1}\left(E_{1}, T_{1}\right)$ is stable, $E(t) \geq 0, T(t) \geq 0$ for every $t \geq 0$.

(ii) The Solution $(E(t), T(t))$ Intersects Line K Infinite Times. Suppose that solution $(E(t), T(t))$ intersects with line $K: T=$ $H$ at times $t_{1}, t_{2}, t_{3}, \ldots, t_{k}, \ldots$, so that $\lim _{k \rightarrow \infty} t_{k}=\infty$. If the conclusion of Lemma 4 is not true, then there is a $t^{*}>t_{0}$ such that $\min \left\{E\left(t^{*}\right), T\left(t^{*}\right)\right\}<0$, and $E(t)>0, T(t)>0$ for every $t_{0} \leq t<t^{*}$. For this $t^{*}$, there is a positive integer $n$ such that $t_{n-1} \leq t^{*} \leq t_{n}$. (i) If $E\left(t^{*}\right)<0, T\left(t^{*}\right) \geq 0$, then from the first and third equations of model (4), we obtain

$$
\begin{aligned}
E\left(t^{*}\right) & \geq(1-p)^{n-1} E\left(t_{0}\right) \exp \left\{\int_{t^{0}}^{t_{*}}[\omega T(s)-\delta] \mathrm{d} s\right\} \\
& \geq 0
\end{aligned}
$$

which leads to a contradiction to $E\left(t^{*}\right)<0$.

(ii) If $T\left(t^{*}\right)<0, E\left(t^{*}\right) \geq 0$, then from the second and fourth equations of model (4), we have

$$
T\left(t^{*}\right) \geq \frac{T_{0}}{\left(1-\beta T_{0}\right) e^{-\alpha t^{*}}+\beta T_{0}} \geq 0,
$$

which leads to a contradiction to $T\left(t^{*}\right)<0$.

So we know that $t^{*}$ does not exist such that $\min \left\{E\left(t^{*}\right)\right.$, $\left.T\left(t^{*}\right)\right\}<0$. Therefore, we can prove that $E(t) \geq 0, T(t) \geq 0$ for every $t \geq 0$.

Next, for the convenience of statement in the remainder of this paper, we introduce the following definitions. Let $\Gamma$ be an arbitrary set in $\mathbb{R}^{2}$ and $Y$ be an arbitrary point in $\mathbb{R}^{2}$. The distance between $Y$ and $\Gamma$ is defined by $d(Y, \Gamma)=\inf _{Y_{0} \in \Gamma} \mid Y-$ $Y_{0} \mid$.

Definition 5 (orbital stability [15]). Trajectory $O^{+}\left(X_{0}, t_{0}\right)$ is said to be orbitally stable if, given $\varepsilon>0$, there exists a constant $\delta=\delta(\varepsilon)>0$ such that, for any solution $X^{*}(t)$ of model (4), when $d\left(X^{*}\left(t_{0}\right), O^{+}\left(X_{0}, t_{0}\right)\right)<\delta$, one has $d\left(X^{*}(t), O^{+}\left(X_{0}, t_{0}\right)\right)<\varepsilon$ for all $t>t_{0}$.

Definition 6 (orbitally asymptotical stability [15]). Trajectory $\mathrm{O}^{+}\left(X_{0}, t_{0}\right)$ is said to be orbitally asymptotically stable if it is orbitally stable; there exists a constant $\eta>0$ such that, for any other solution $X^{*}(t)$ of model (4), when $d\left(X^{*}\left(t_{0}\right)\right.$, $\left.O^{+}\left(X_{0}, t_{0}\right)\right)<\eta$, then $\lim _{t \rightarrow \infty} d\left(X^{*}(t), O^{+}\left(X_{0}, t_{0}\right)\right)=0$.

To analyze the dynamic behaviors of model (4), we define two sections to the phase space of model (4) by

$$
\begin{aligned}
\Gamma_{q} & :=\{(E, T): E \geq 0, T=(1-q) H\}, \\
\Gamma_{H} & :=\{(E, T): E \geq 0, T=H\} .
\end{aligned}
$$

For any point $a_{n}\left(E_{n}, H\right)$ on section $\Gamma_{H}$, suppose that trajectory $\mathrm{O}^{+}\left(a_{n}, t_{n}\right)$ starting from point $a_{n}$ intersects section $\Gamma_{H}$ infinitely many times. That is, trajectory $\mathrm{O}^{+}\left(a_{n}, t_{n}\right)$ jumps to point $a_{n}^{+}\left((1-p) E_{n}+\tau,(1-q) H\right)$ on section $\Gamma_{q}$ due to impulsive effects $\Delta E(t)=-p E\left(t^{-}\right)+\tau$ and $\Delta T(t)=-q H$. Furthermore, trajectory $\mathrm{O}^{+}\left(a_{n}, t_{n}\right)$ intersects section $\Gamma_{H}$ at point $a_{n+1}\left(E_{n+1}, H\right)$ and then jumps to point $a_{n+1}^{+}\left((1-p) E_{n+1}+\right.$ $\tau,(1-q) H$ ) on section $\Gamma_{q}$ again (see Figure $\left.1(\mathrm{~b})\right)$. Repeating this process, we have two impulsive point sequences $\left\{a_{n}^{+}((1-\right.$ $\left.\left.p) E_{n}+\tau,(1-q) H\right)\right\}$ and $\left\{a_{n}\left(E_{n}, H\right)\right\}$, where $E_{n+1}$ is determined by $E_{n}, p, q, \tau, H$ and $n=1,2, \ldots$. We then define the Poincaré map of section $\Gamma_{H}$ as follows:

$$
E_{n+1}=P\left(E_{n}, p, q, \tau, H\right) .
$$




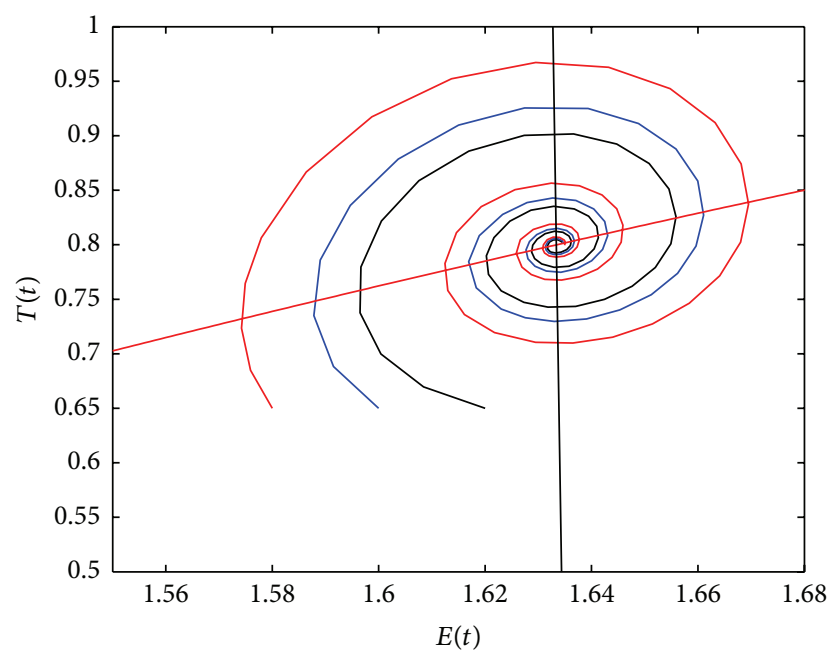

(a)

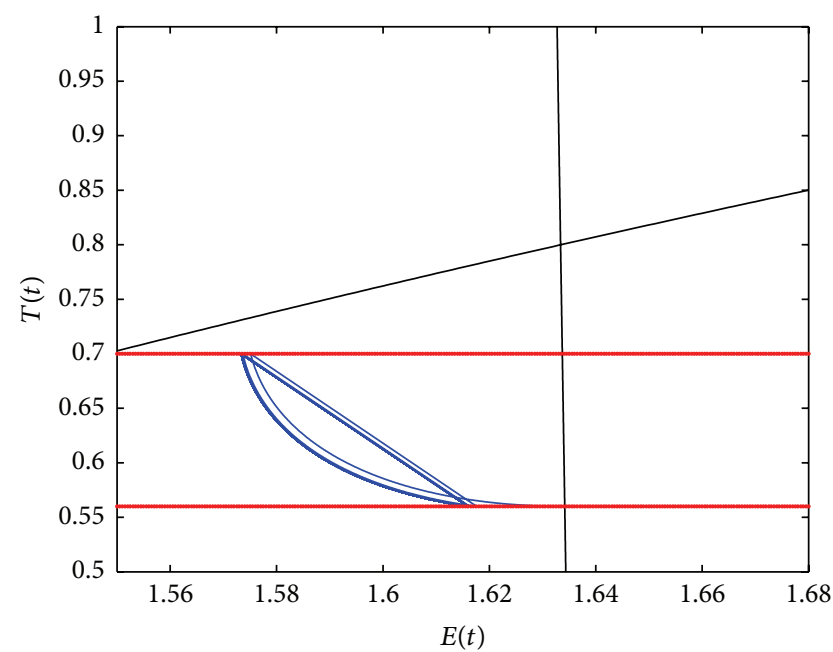

(b)

FIgURE 1: The difference of dynamical behaviors for models (1) and (4).

Definition 7. A trajectory $\mathrm{O}^{+}\left(a_{n}, t_{n}\right)$ of model (4) is said to be order- $k$ periodic if there exists a positive integer $k \geq 1$ such that $k$ is the smallest integer for $E_{n+k}=E_{n}$.

Next, we consider the following autonomous model with impulsive effects:

$$
\begin{aligned}
& \frac{\mathrm{d} x}{\mathrm{~d} t}=f(x, y), \\
& \frac{\mathrm{d} y}{\mathrm{~d} t}=g(x, y) \text {, } \\
& \varphi(x, y) \neq 0, \\
& \Delta x=\xi(x, y) \text {, } \\
& \Delta y=\eta(x, y), \\
& \varphi(x, y)=0,
\end{aligned}
$$

$$
\begin{aligned}
\mu & =\prod_{j=1}^{n} \kappa_{j} \\
& \cdot \exp \left\{\int_{0}^{T}\left[\frac{\partial f(\mu(t), \nu(t))}{\partial x}+\frac{\partial g(\mu(t), \nu(t))}{\partial y}\right] \mathrm{d} t\right\}
\end{aligned}
$$

with

$$
\kappa_{j}=\frac{((\partial \eta / \partial y)(\partial \varphi / \partial x)-(\partial \eta / \partial x)(\partial \varphi / \partial y)+\partial \varphi / \partial x) f_{+}+((\partial \xi / \partial x)(\partial \varphi / \partial y)-(\partial \xi / \partial y)(\partial \varphi / \partial x)+\partial \varphi / \partial y) g_{+}}{(\partial \varphi / \partial x) f+(\partial \varphi / \partial y) g}
$$

\section{Main Results}

The first result is on the existence of a positive order-1 periodic solution for model (4).
Theorem 9. For any $q, p \in(0,1)$, model (4) admits a positive order-1 periodic solution.

Proof. Let the point $c_{1}(\varepsilon,(1-q) H) \in \Gamma_{q}$ for sufficiently small $\varepsilon$ with $\varepsilon \leq \tau$. In view of the geometrical structure of the phase space of model (4), trajectory $\mathrm{O}^{+}\left(c_{1}, t_{0}\right)$ of model (4) starting from initial point $c_{1}$ intersects section $\Gamma_{H}$ at point $d_{1}\left(E_{1}, H\right)$. At point $d_{1}$, the trajectory $O^{+}\left(c_{1}, t_{0}\right)$ jumps to point $c_{2}\left((1-p) E_{1}+\tau,(1-q) H\right)$ on section $\Gamma_{q}$ due to impulsive effects $\Delta E(t)=-p E\left(t^{-}\right)+\tau$ and $\Delta T(t)=-q T\left(t^{-}\right)$. Furthermore, the trajectory $O^{+}\left(c_{1}, t_{0}\right)$ intersects section $\Gamma_{H}$ at point $d_{2}\left(E_{2}, H\right)$. 
Since $\varepsilon \leq \tau$, then $\varepsilon<(1-p) E_{1}+\tau$. It follows that $c_{1}$ is on the left of $c_{2}$. We claim that $d_{1}$ is also on the left of $d_{2}$. In fact, if $d_{1}$ is on the right of $d_{2}$, then orbits $\widetilde{c_{1} d_{1}}$ and $\widetilde{c_{2} d_{2}}$ intersect at the point $\left(E_{0}, T_{0}\right)$. This shows that there are two different solutions which start from the point $\left(E_{0}, T_{0}\right)$. This contradicts with the uniqueness of solutions for model (4). So by (8), we have $E_{2}=P\left(E_{1}, p, q, \tau, H\right)$ and

$$
P\left(E_{1}, p, q, \tau, H\right)-E_{1}=E_{2}-E_{1}>0 .
$$

On the other hand, suppose that the curve $l_{1}: \alpha-\alpha \beta T-$ $E=0$ intersects section $\Gamma_{q}$ at point $a_{0}(\alpha-(1-q) \alpha \beta H,(1-q) H)$. Then trajectory $\mathrm{O}^{+}\left(a_{0}, t_{0}\right)$ starting from the initial point $a_{0}$ intersects section $\Gamma_{H}$ at point $b_{1}\left(\widehat{E}_{1}, H\right)$ and then jumps to point $a_{1}\left((1-p) \widehat{E}_{1}+\tau,(1-q) H\right)$ on section $\Gamma_{q}$ and finally reaches a point $b_{2}\left(\widehat{E}_{2}, H\right)$ in section $\Gamma_{H}$ again. If there is a positive constant $\tau^{*}$ such that $(1-p) \widehat{E}_{1}+\tau^{*}=\alpha-(1-q) \alpha \beta H$, then $a_{1}$ coincides with $a_{0}$ for $\tau=\tau^{*} \in(0,1)$. That is, $b_{1}$ coincides with $b_{2}$. Otherwise, $a_{1}$ is on the left of $a_{0}$ for $(1-p) \widehat{E}_{1}+\tau<\alpha-(1-q) \alpha \beta H$ and $a_{1}$ is on the right of $a_{0}$ for $(1-p) \widehat{E}_{1}+\tau>\alpha-(1-q) \alpha \beta H$. However, from the geometrical structure of a phase space of model $(4), b_{2}$ is on the left of $b_{1}$ for any $\tau \in\left(0, \tau^{*}\right) \cup\left(\tau^{*},+\infty\right)$.

To sum up, we get, from the above discussions, that

(i) when $\widehat{E}_{1}=\widehat{E}_{2}$, model (4) has a positive order-1 periodic solution;

(ii) when $\widehat{E}_{2}<\widehat{E}_{1}$,

$$
P\left(\widehat{E}_{1}, p, q, \tau, H\right)-\widehat{E}_{1}=\widehat{E}_{2}-\widehat{E}_{1}<0 .
$$

By (12) and (13), it follows that the Poincaré map (8) has a fixed point. This means that model (4) has a positive order-1 periodic solution. The proof is complete.

The next result is on the orbital stability of a positive order-1 periodic solution for model (4).

Theorem 10. Let $(\phi(t), \psi(t))$ be a positive order-1 periodic solution of model (4) with period $\omega$. If

$$
|\mu|=|\kappa| \exp \left\{\int_{0}^{\infty}[\omega \psi(t)-\delta] \mathrm{d} t\right\}<1,
$$

where

$\kappa$

$$
=\frac{(1-p)(1-q)[\alpha-\alpha \beta(1-q) H-(1-p) \phi(\varpi)-\tau]}{\alpha-\alpha \beta H-\phi(\varpi)},
$$

then $(\phi(t), \psi(t))$ is locally orbitally asymptotically stable.

Proof. Suppose that $(\phi(t), \psi(t))$ intersects sections $\Gamma_{p}$ and $\Gamma_{H}$ at points $K^{+}((1-p) \phi(\varpi)+\tau,(1-p) H)$ and $E(\phi(\varpi), H)$, respectively. Comparing with model (9), we have

$$
\begin{aligned}
& f(E, T)=\sigma+\omega T E-\delta E, \\
& g(E, T)=\alpha T(1-\beta T)-T E,
\end{aligned}
$$

and $\xi(E, T)=-p E+\tau, \eta(E, T)=-q T, \varphi(E, T)=T-H$, $(\phi(@), \psi(@))=(\phi(@), H)$, and $\left(\phi\left(T^{*+}\right), \psi\left(T^{*+}\right)\right)=((1-$ p) $\phi(\varpi)+\tau,(1-q) H)$. Thus

$$
\begin{aligned}
& \frac{\partial f}{\partial E}=\omega T-\delta, \\
& \frac{\partial g}{\partial T}=\alpha-2 \alpha \beta T-E, \\
& \frac{\partial \xi}{\partial E}=-p, \\
& \frac{\partial \eta}{\partial T}=-q, \\
& \frac{\partial \varphi}{\partial T}=1, \\
& \frac{\partial \xi}{\partial T}=\frac{\partial \eta}{\partial E}=\frac{\partial \varphi}{\partial E}=0 .
\end{aligned}
$$

Furthermore, it follows from (17) that

$$
\begin{aligned}
\kappa & =\frac{((\partial \eta / \partial T)(\partial \varphi / \partial E)-(\partial \eta / \partial E)(\partial \varphi / \partial T)+\partial \varphi / \partial E) f_{+}+((\partial \xi / \partial E)(\partial \varphi / \partial T)-(\partial \xi / \partial T)(\partial \varphi / \partial E)+\partial \varphi / \partial T) g_{+}}{(\partial \varphi / \partial E) f+(\partial \varphi / \partial I) g} \\
& =\frac{(1-p) g_{+}\left(\phi\left(T^{*+}\right), \psi\left(T^{*+}\right)\right)}{g(\phi(\varpi), \psi(\varpi))}=\frac{(1-p)(1-q)[\alpha-\alpha \beta(1-q) H-(1-p) \phi(\varpi)-\tau]}{\alpha-\alpha \beta H-\phi(\varpi)}, \\
\mu & =\kappa \exp \left\{\int_{0}^{T}[\omega \psi(t)-\delta+\alpha-2 \alpha \beta \psi(t)-\phi(t)] \mathrm{d} t\right\} .
\end{aligned}
$$


On the other hand, integrating both sides of the second equation of model (4) along the orbit $\widetilde{c^{+} c}$, we have

$$
\begin{aligned}
\ln \frac{1}{1-q} & =\int_{(1-q) H}^{H} \frac{\mathrm{d} T}{T}=\int_{0}^{T^{*}}[\alpha-2 \alpha \beta T-E] \mathrm{d} t \\
& =\int_{0}^{\oplus}[\alpha-2 \alpha \beta \psi(t)-\phi(t)] \mathrm{d} t .
\end{aligned}
$$

From (18)-(19), we can obtain

$$
\begin{aligned}
& |\mu| \\
& =\left|\frac{(1-p)(1-q)[\alpha-\alpha \beta(1-q) H-(1-p) \phi(\omega)-\tau]}{\alpha-\alpha \beta H-\phi(\omega)}\right| \\
& =\frac{1}{1-q} \exp \left\{\int_{0}^{\infty}[\omega \psi(t)-\delta] \mathrm{d} t\right\} \\
& =\left|\frac{(1-p)[\alpha-\alpha \beta(1-q) H-(1-p) \phi(\omega)-\tau]}{\alpha-\alpha \beta H-\phi(\omega)}\right| \\
& \cdot \exp \left\{\int_{0}^{\infty}[\omega \psi(t)-\delta] \mathrm{d} t\right\} .
\end{aligned}
$$

By condition (14), we see that model (4) satisfies all conditions of Lemma 8 . It then follows from Lemma 8 that the order-1 periodic solution $(\phi(t), \psi(t))$ of model (4) is locally orbitally asymptotically stable and has asymptotic phase property. This completes the proof.

From the proof of Theorem 10, integrating both sides of the first equation of model (4) along orbit $\widetilde{c^{+} c}$, it follows that

$$
\begin{aligned}
& \exp \left\{\int_{0}^{\omega}[\omega \psi(t)-\delta] \mathrm{d} t\right\} \leq \exp \left\{\int_{0}^{\omega}(\omega H-\delta) \mathrm{d} t\right\} \\
& \quad \leq \exp \{(\omega H-\omega) \omega\} .
\end{aligned}
$$

Therefore, we have the consequence of Theorem 10 as Corollary 11 below.

Corollary 11. Let $(\phi(t), \psi(t))$ be a positive order-1 periodic solution of model (4) with periodic $\omega$. If

$$
\begin{aligned}
& \qquad \mu \mid \\
& =\left|\frac{(1-p)[\alpha-\alpha \beta(1-q) H-(1-p) \phi(\varpi)-\tau]}{\alpha-\alpha \beta H-\phi(\varpi)}\right| \\
& \cdot \exp \{(\omega H-\delta) \varpi\}<1, \\
& \text { then }(\phi(t), \psi(t)) \text { is locally orbitally asymptotically stable. }
\end{aligned}
$$

Theorem 12. If $\tau<\alpha-\alpha \beta(1-q) H$, then model (4) has a positive order-1 periodic solution which is orbitally asymptotical stable.

Proof. Suppose that the curve $l_{1}: \alpha-\alpha \beta T-E=0$ intersects section $\Gamma_{q}$ at point $a_{0}(\alpha-(1-q) \alpha \beta H,(1-q) H)$. Let $\alpha-(1-$ q) $\alpha \beta H=\lambda_{0}$. Then, since $\tau<\lambda_{0}$, there is a positive constant $\lambda^{*}$ such that $(1-p) \lambda^{*}+\tau=\lambda_{0}$. For any two points $a_{i}\left(E_{i}, H\right)$ and $a_{j}\left(E_{j}, H\right)$ on section $\gamma_{H}, E_{i}, E_{j} \in\left(0, \lambda^{*}\right)$ and $E_{i}<E_{j}$. In view of the impulsive treatment effects, point $a_{i}^{+}\left((1-p) E_{i}+\right.$ $\tau,(1-q) H)$ is on the left of point $a_{j}^{+}\left((1-p) E_{j}+\tau,(1-q) H\right)$. Therefore, from the geometrical construction of the phase space of model (4) we have

$$
E_{i+1}<E_{j+1}<\lambda^{*} .
$$

Then, for any $E_{0} \in\left(0, \lambda^{*}\right)$, from the Poincaré map (8) of section $\Gamma_{H}$ we have $E_{1}=F\left(E_{0}, p, q, \tau, H\right), E_{2}=F\left(E_{1}, p, q, \tau\right.$, $H)$, and $E_{n+1}=F\left(E_{n}, p, q, \tau, H\right)(n=2,3, \ldots)$. In particular, if $E_{0}=E_{1}$, then model (4) has a positive order-1 periodic solution.

Next, we discuss the general circumstance; that is, $E_{0} \neq$ $E_{1} \neq E_{2} \neq E_{3} \neq \cdots \neq E_{k}(k \geq 2)$.

(1) If $E_{1}<E_{0}$, then it follows that $E_{2}<E_{1}, E_{3}<E_{2}$. Repeating the above process, we have

$$
0<\cdots<E_{5}<E_{4}<E_{3}<E_{2}<E_{1}<E_{0} .
$$

(2) If $E_{0}<E_{1}$, then it follows that $E_{1}<E_{2}, E_{2}<E_{3}$. Repeating the above process, we have

$$
E_{0}<E_{1}<E_{2}<E_{3}<E_{4}<E_{5}<\cdots<\lambda^{*} .
$$

Further in Case (1) it follows $\lim _{k \rightarrow \infty} E_{k}=\delta_{1}$, where $0<\delta_{1}<E_{0}$. Similarly, in Case (2) $\lim _{k \rightarrow \infty} S_{k}=\delta_{2}$, where $S_{0}<\delta_{2}<\lambda^{*}$. So model (4) has orbitally asymptotically stable positive order-1 periodic solution.

Remark 13. Obviously, the treatment strength $p$ of chemotherapy is closely related to the stable region of an order1 solution. If $p$ is close to 1 , then $\lambda^{*}$ is large, so by Theorem 12 , the local asymptotic orbital stability region is large. Otherwise, if $p$ is close to 0 , then the local asymptotic orbital stability region is small. That is, the local asymptotic orbital stability region depends on value of $p$.

Remark 14. We study the existence and the stability of a positive order-1 periodic solution for the case (ii) of Lemma 1. For the case (ii) of Lemma 1, model (4) has an unstable equilibrium $P_{0}$ and a stable positive equilibrium $P_{1}$, so that we can obtain similar results of case (ii) of Lemma 1 by using the same methods. Therefore, we only consider numerical simulations for case (iii) of Lemma 1 in next section.

\section{Numerical Results}

In this work, we investigate a tumor-immune model with state-dependent impulsive immunotherapy and chemotherapy. By the Poincaré map, analogue of Poincaré criterion, and qualitative analysis method, we give the criteria for the existence and stability of the positive periodic solution for this model. In order to illustrate the implications of our results and the feasibility of the state-dependent pulse feedback control strategy, we consider a tumor-immune model with state-dependent impulsive immunotherapy and chemotherapy. For simulation, we use parameter values in Table 1.

Firstly, it is easy to obtain that the model (4) without impulsive effects has a stable focus $(0.8333,3.1667)$. The plots 


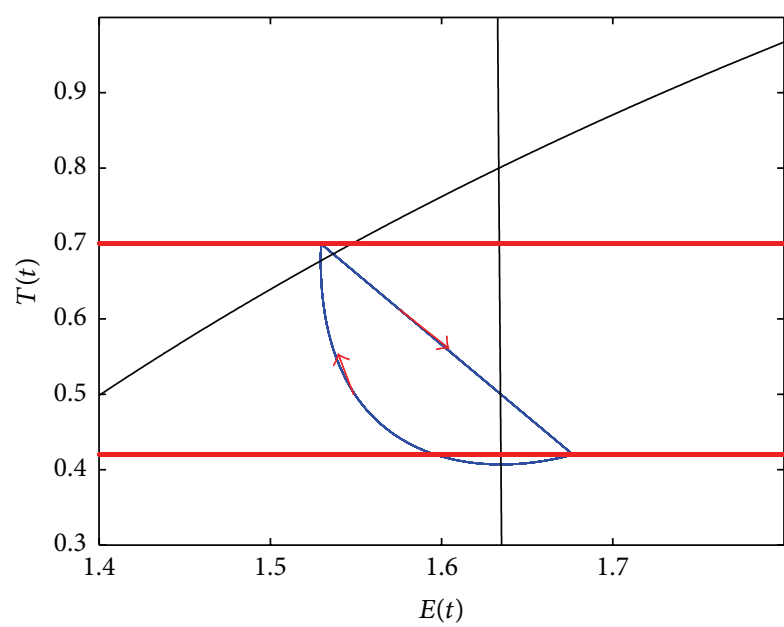

(a)

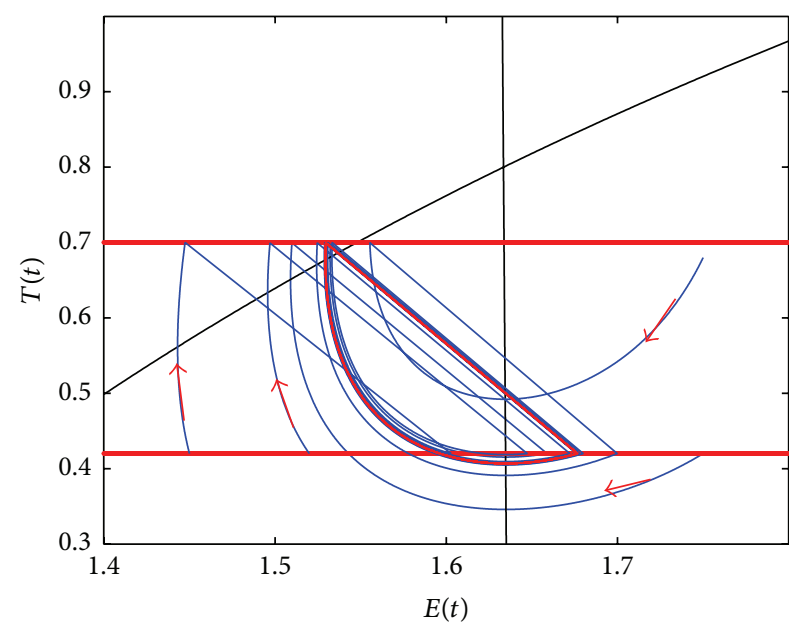

(b)

FIGURE 2: The existence and stability of order-1 periodic solution for model (4) with $p=0.1, q=0.4$, and $\tau=0.2$ : (a) the existence; (b) the orbital asymptotical stability.

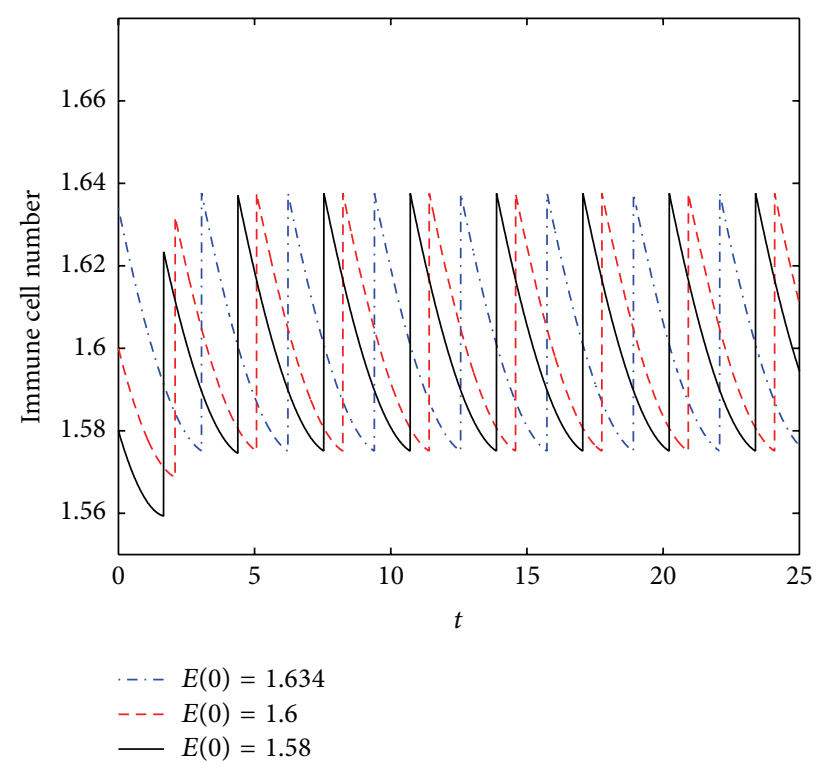

(a)

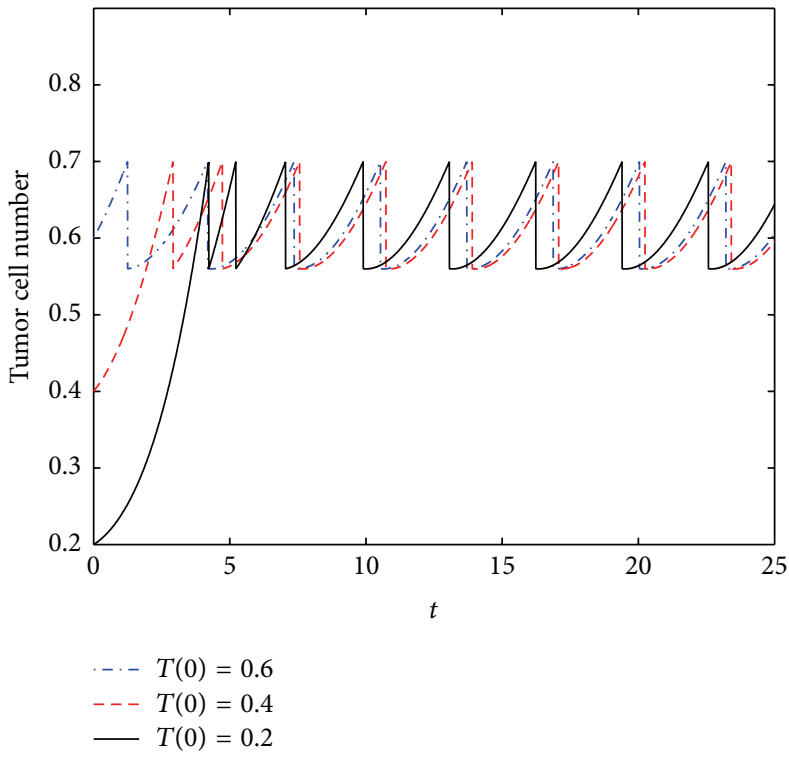

(b)

FIgURE 3: The existence and stability of positive order-1 periodic solution of model (4) for case (iii) in Lemma 1.

in Figure 1(a) show the tumor-immune dynamical behaviors in patients without therapies, which implies that cancer is deadly. Now, we apply chemotherapy and immunotherapy to control tumor cells. That is, we choose control parameters $p=0.1, q=0.2, \tau=0.22$, and $H=0.7$; the plots in Figure 1(b) show that model (4) has a positive order-1 periodic solution. This indicates that tumor cells might be controlled below the threshold value by state-dependent impulsive control strategies.

Nextly, we choose control parameters $p=0.1, q=0.4$, $\tau=0.3$, and $H=0.7$ and other model parameters are fixed in Table 1. From Theorem 9, model (4) admits a positive order-1 periodic solution $(\phi(t), \psi(t))$ starting from point
$(1.6767,0.42) \in \Gamma_{p}$ and intersection section $\Gamma_{H}$ at point $(1.5296,0.7)=(\phi(T), \psi(T))$. This is shown in Figure 2(a). Further, this can be easily calculated by Corollary 11:

$$
\begin{aligned}
& |\mu| \\
& =\left|\frac{(1-p)[\alpha-\alpha \beta(1-q) H-(1-p) \phi(T)-\tau]}{\alpha-\alpha \beta H-\phi(T)}\right| \\
& \cdot \exp \{\omega H T\} \approx 0.4753<1 .
\end{aligned}
$$

This shows that the positive order-1 periodic solution is orbitally asymptotically stable. This is shown in Figure 2(b). The plots in Figures 3(a) and 3(b) show that the existence and 
TABLE 1: Equations and parameter descriptions.

\begin{tabular}{cccc}
\hline State & Term & Parameter & Description \\
\hline \multirow{2}{*}{$E(t)$} & $\sigma$ & $\sigma=0.1181[17]$ & Production of effect cell \\
& $\omega T E$ & $\omega=0.04[18]$ & Stimulated accumulation of effect cell by tumor cell \\
& $\delta E$ & $\alpha=1$ (assumed) & Turnover of effect cell \\
\hline$T(t)$ & $\alpha T(1-\beta T)$ & Tumor growth term \\
& $T E$ & & Effect cell induced tumor death \\
\hline
\end{tabular}
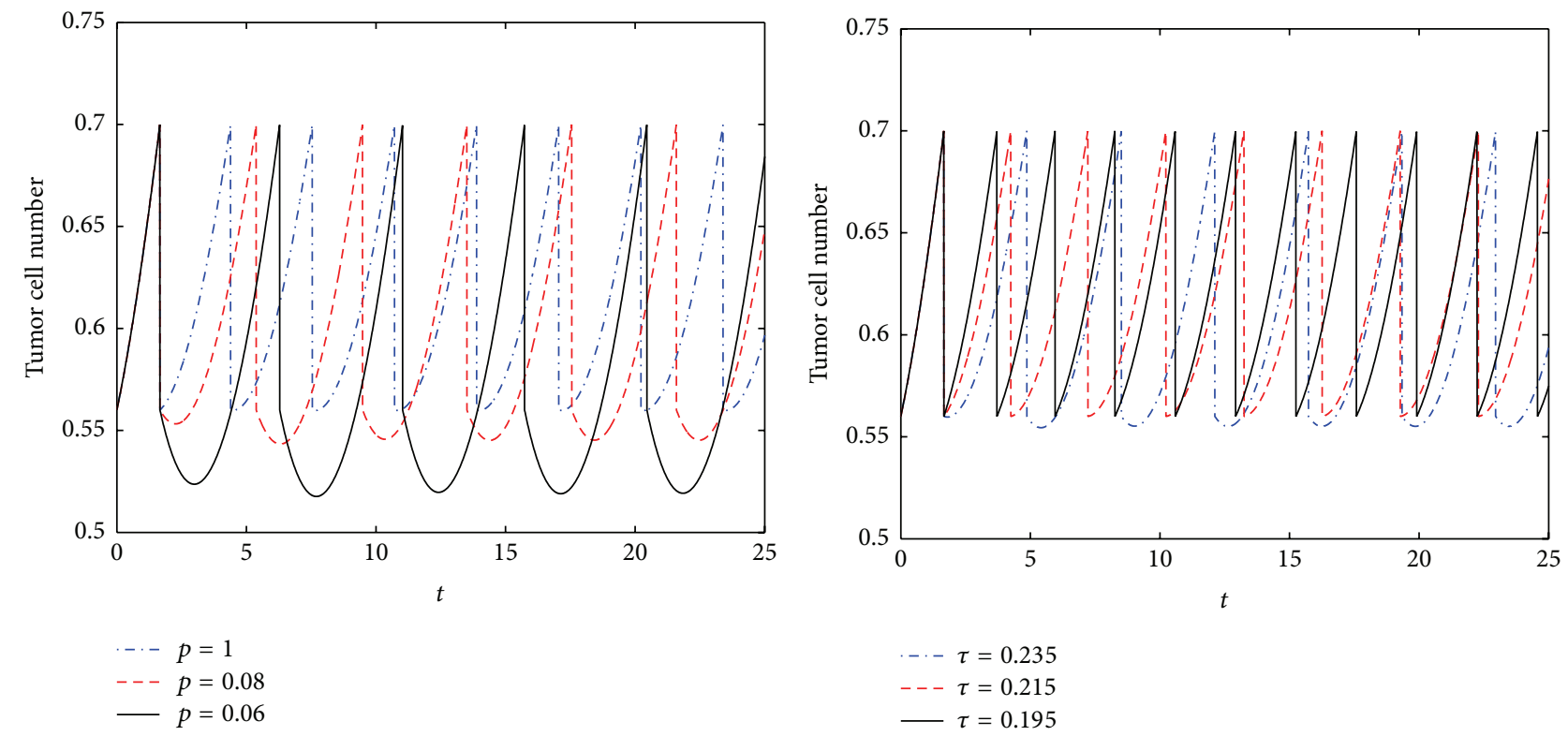

(a)

(b)

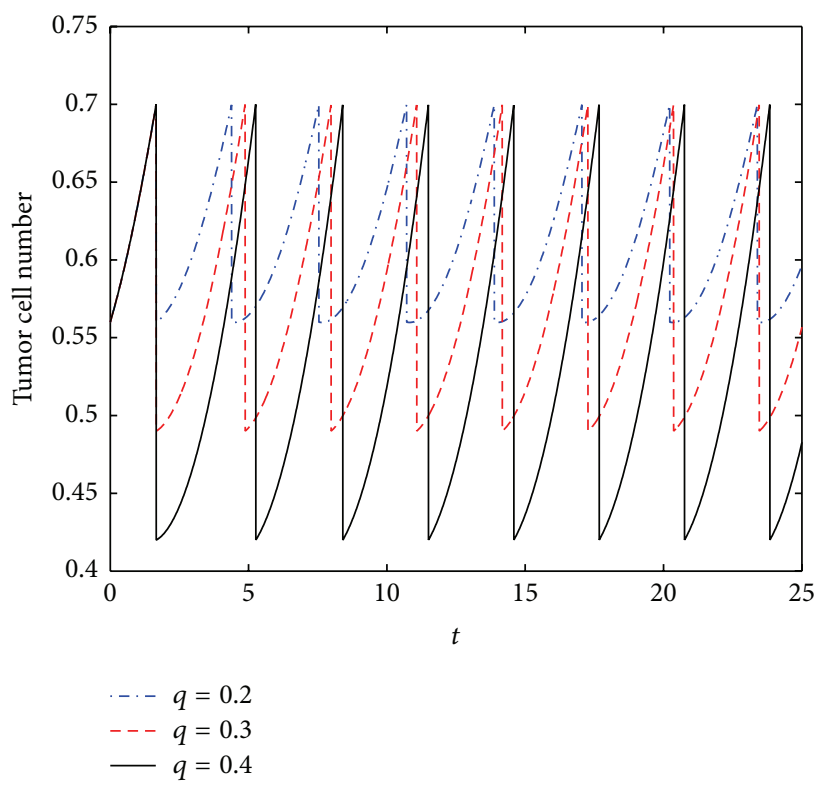

(c)

Figure 4: The effect of control parameters $p, q$, and $\tau$ for the dynamical behaviors of model (4): (a) $p=0.1,0.08$, and 0.06 , respectively; (b) $\tau=0.235,0.215$, and 0.195 , respectively; (c) $q=0.2,0.3$, and 0.4 , respectively. 
stability of an order-1 periodic solution of model (4) for case (iii) in Lemma 1.

Thirdly, we investigate how the strength of chemotherapy and immunotherapy affects the control of tumor cell numbers and existence and stability of the periodic solutions. The value $p$ means the side effects of chemotherapy to immune cells. Now, we change only the values of $p$ such that $0.06,0.08,0.1$ but the other parameter values are fixed. In Figure 3(a), our simulation results show that if the value of $p$ increases, then periodic $T$ of positive order-1 periodic solution for model (4) decreases. In particular, if $p=0.06$, then we can see that the tumor cell numbers fall off more than the other cases. Secondly, let $\tau$ denote amounts of injected immune cells for immunotherapy. In Figure 3(b), we can see that the periodic $T$ of an order-1 periodic solution decreases with decreasing value of $\tau$. The fourth injection that the value of $\tau$ is 0.235 and the fifth injection that the value of $\tau$ is 0.195 happen simultaneously. Thirdly, the value $q$ implies the strength of chemotherapy to tumor cells. Then there are some differences of simulation results between the value $q$ and the values $p$ and $\tau$. Numerical simulation shows that the period $T$ of positive order-1 periodic solution for model (4) increases with the increase of the strength of chemotherapy to tumor cell $q$ (see Figure 3(c)). And if the value of $p$ increases, tumor cell's lower bounded line is lowered in Figure 4(a). These show that the recurrence time for tumor cells can be prolonged by increasing strength of chemotherapy to tumor cells $q$, amounts of injected immune cells $\tau$, and decreasing side effects of chemotherapy to immune cells $p$ by through simulation results (see Figures 4(b) and 4(c)).

Finally, we investigate the effect of the model with two types of controls: fixed-time dependent impulsive control and state-dependent impulsive control. We first consider two treatment (or injection) period scenarios (the periods $T=$ $1.665,2.96)$ for this model with fixed-time dependent impulsive control and $p=0.1, q=0.2, \tau=0.22, E(0)=1.58$, and $T(0)=0.56$ as other parameters are fixed in Table 1 . When period $T=1.665$, the tumor cells are controlled in lower than the hazardous threshold value $H=0.7$ which is shown in Figure 5 by blue line. However, if period $T=2.96$, the tumor control fails. That is, the number of tumor cells is higher than the hazardous threshold value $H=0.7$ which is shown in Figure 5 by red line. On the other hand, model (4) with statedependent impulsive control with threshold value $H=0.7$ has approximately $T=3.0$ as treatment period and we can control the number of tumor cells in the prescribed fixed boundary which is shown in Figure 5 by black line. As we know, the proper treatment schedule is very important to control tumor cells because of the costs for treatment.

\section{Conclusion}

In this paper, a mathematical model of tumor and immune cells with state-dependent impulsive chemotherapy and immunotherapy was considered. This is the first attempt to investigate a tumor-immune model with state-dependent impulsive treatments. The criterion for the existence and stability of the positive periodic solution of tumor-immune

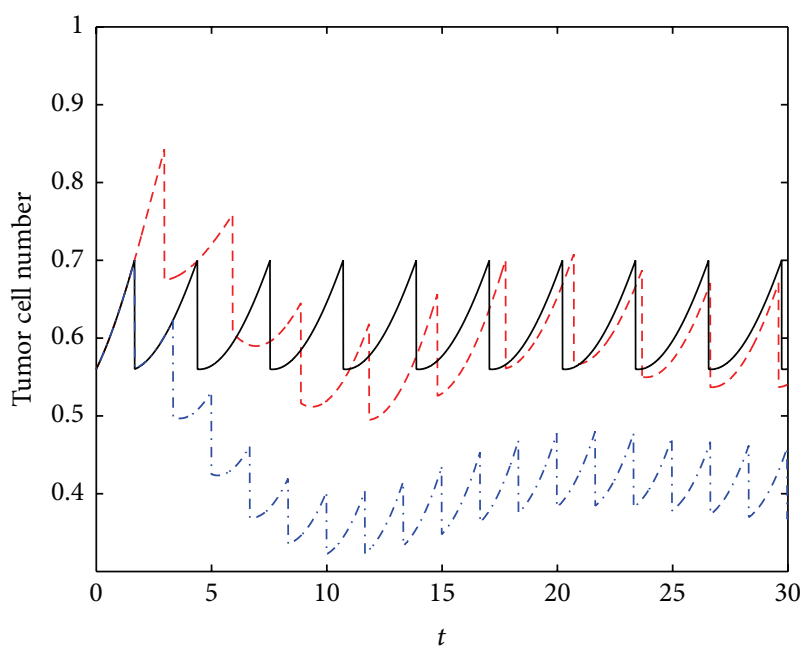

- - Fixed-time impulsive control $(t=2.96)$

- State-dependent impulsive control

. - - Fixed-time impulsive control $(t=1.665)$

FIGURE 5: The comparison of the fixed-time impulsive effect and the state impulsive effect $(p=0.1, q=0.2, \tau=0.22, E(0)=1.58$, and $T(0)=0.56)$.

model, based on Poincaré map and analogue of Poincaré criterion, was presented. We proved existence of positive order-1 periodic solutions and obtained condition of orbitally asymptotical stability of positive order-1 periodic solution for our model. From the results, we were able to see that local asymptotic orbital stability region is large as treatment strength $p$ of chemotherapy is increasing. Also, increasing strength of chemotherapy and immunotherapy and decreasing side effects of chemotherapy lengthened tumor recurrence time. The simulation results indicate that in determining optimal treatment timing the model with state-dependent impulsive control is more efficient than fixed-time impulsive control.

\section{Competing Interests}

The authors declare that there is no conflict of interests regarding the publication of this paper.

\section{Acknowledgments}

The authors would like to thank antonymous referees for their constructive suggestions and comments that improve substantially the original manuscript. This research was supported by Basic Science Research Program through the National Research Foundation of Korea (NRF) funded by the Ministry of Education, Science and Technology (NRF2015R1D1A1A02062131). This work was supported in part by the National Natural Science Foundation of China (Grant no. 11461067) and the Natural Science Foundation of Xinjiang (Grant no. 2016D01C046).

\section{References}

[1] K. S. Kim, G. Cho, and I. H. Jung, "Optimal treatment strategy for a tumor model under immune suppression," Computational 
and Mathematical Methods in Medicine, vol. 2014, Article ID 206287, 13 pages, 2014.

[2] D. Kirschner and J. C. Panetta, "Modeling immunotherapy of the tumor-immune interaction," Journal of Mathematical Biology, vol. 37, no. 3, pp. 235-252, 1998.

[3] V. A. Kuznetsov, I. A. Makalkin, M. A. Taylor, and A. S. Perelson, "Nonlinear dynamics of immunogenic tumors: parameter estimation and global bifurcation analysis," Bulletin of Mathematical Biology, vol. 56, no. 2, pp. 295-321, 1994.

[4] M. Gałach, "Dynamics of the tumor-immune system competition - the effect of time delay," International Journal of Applied Mathematics and Computer Science, vol. 13, no. 3, pp. 395-406, 2003.

[5] D. Dingli, M. D. Cascino, K. Josić, S. J. Russell, and Ž. Bajzer, "Mathematical modeling of cancer radiovirotherapy," Mathematical Biosciences, vol. 199, no. 1, pp. 55-78, 2006.

[6] X. Z. Meng and L. S. Chen, "Global dynamical behaviors for an SIR epidemic model with time delay and pulse vaccination," Taiwanese Journal of Mathematics, vol. 12, no. 5, pp. 1107-1122, 2008.

[7] Y. Z. Pei, S. Y. Liu, C. G. Li, and L. S. Chen, "The dynamics of an impulsive delay SI model with variable coefficients," Applied Mathematical Modelling, vol. 33, no. 6, pp. 2766-2776, 2009.

[8] T. Gao, W. Wang, and X. Liu, "Mathematical analysis of an HIV model with impulsive antiretroviral drug doses," Mathematics and Computers in Simulation, vol. 82, no. 4, pp. 653-665, 2011.

[9] M. H. Qiao, H. Qi, and Y. C. Chen, "Qualitative analysis of hepatitis $\mathrm{B}$ virus infection model with impulsive vaccination and time delay," Acta Mathematica Scientia B, vol. 31, no. 3, pp. 1020-1034, 2011.

[10] M. Z. Huang, J. X. Li, X. Y. Song, and H. J. Guo, "Modeling impulsive injections of insulin: towards artificial pancreas," SIAM Journal on Applied Mathematics, vol. 72, no. 5, pp. 15241548, 2012.

[11] S. Tang, Y. Xiao, and D. Clancy, "New modelling approach concerning integrated disease control and cost-effectivity," Nonlinear Analysis: Theory, Methods \& Applications, vol. 63, no. 3, pp. 439-471, 2005.

[12] L. Nie, Z. Teng, and A. Torres, "Dynamic analysis of an SIR epidemic model with state dependent pulse vaccination," Nonlinear Analysis: Real World Applications, vol. 13, no. 4, pp. 16211629, 2012.

[13] L.-F. Nie, Z.-D. Teng, and I. H. Jung, "Complex dynamic behavior in a viral model with state feedback control strategies," Nonlinear Dynamics, vol. 77, no. 4, pp. 1223-1236, 2014.

[14] B. Tang, Y. N. Xiao, S. Y. Tang, and R. A. Cheke, "A feedback control model of comprehensive therapy for treating immunogenic tumours," International Journal of Bifurcation and Chaos, vol. 26, no. 3, Article ID 1650039, 22 pages, 2016.

[15] J. K. Hale and H. Koçak, Dynamics and Bifurcations, Springer, New York, NY, USA, 1991.

[16] P. S. Simeonov and D. D. Bainov, "Orbital stability of periodic solutions of autonomous systems with impulse effect," International Journal of Systems Science, vol. 19, no. 12, pp. 2561-2585, 1988.

[17] L. G. de Pillis, W. Gu, and A. E. Radunskaya, "Mixed immunotherapy and chemotherapy of tumors: modeling, applications and biological interpretations," Journal of Theoretical Biology, vol. 238, no. 4, pp. 841-862, 2006.
[18] L. de Pillis, K. R. Fister, W. Gu et al., "Mathematical model creation for cancer chemo-immunotherapy," Computational and Mathematical Methods in Medicine, vol. 10, no. 3, pp. 165-184, 2009. 


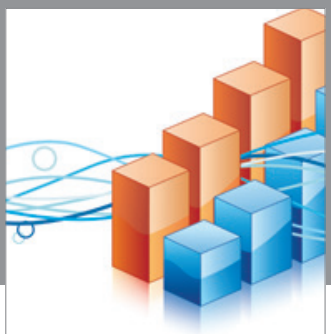

Advances in

Operations Research

vatem alat4

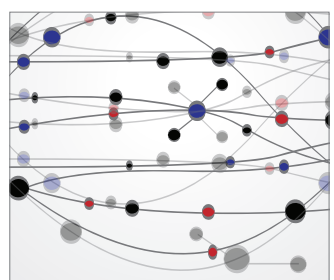

\section{The Scientific} World Journal
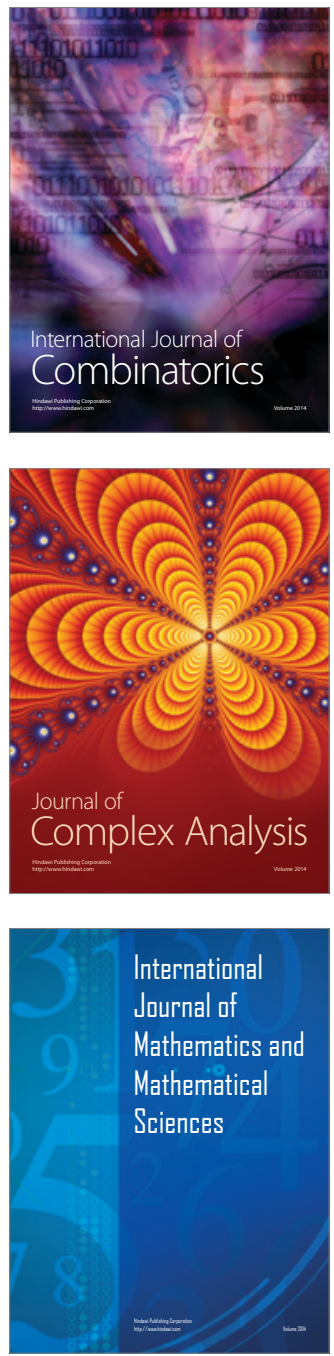
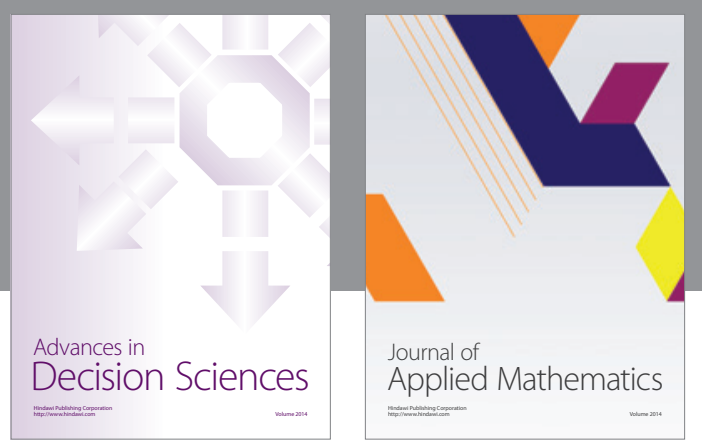

Algebra

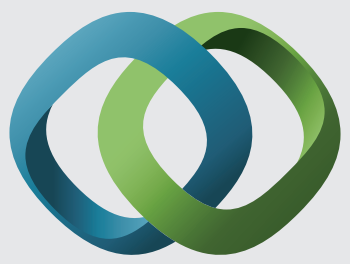

\section{Hindawi}

Submit your manuscripts at

http://www.hindawi.com
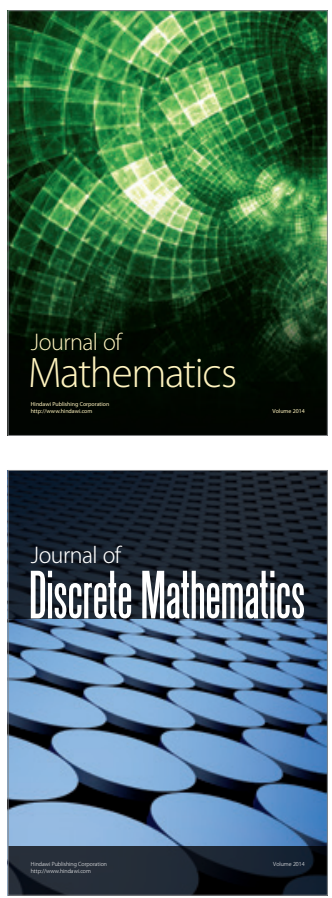

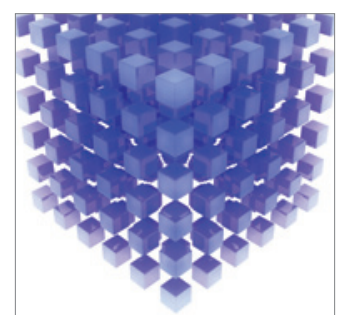

Mathematical Problems in Engineering
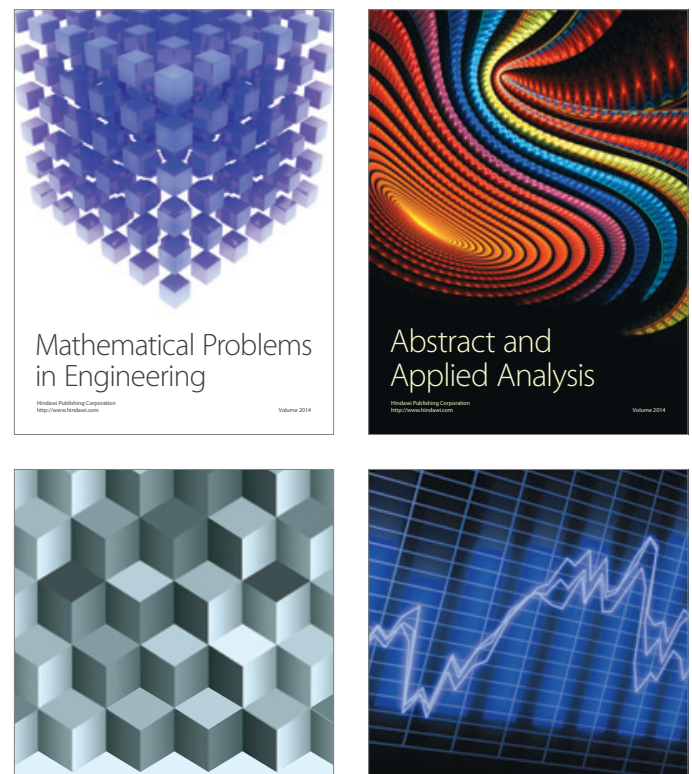

Journal of

Function Spaces

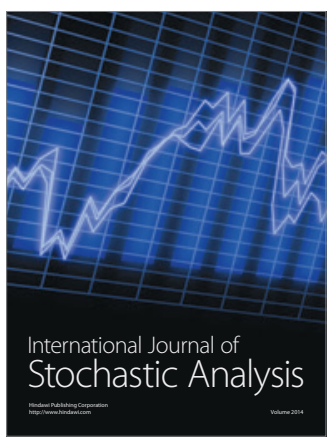

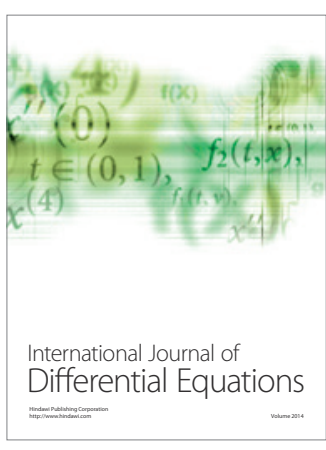
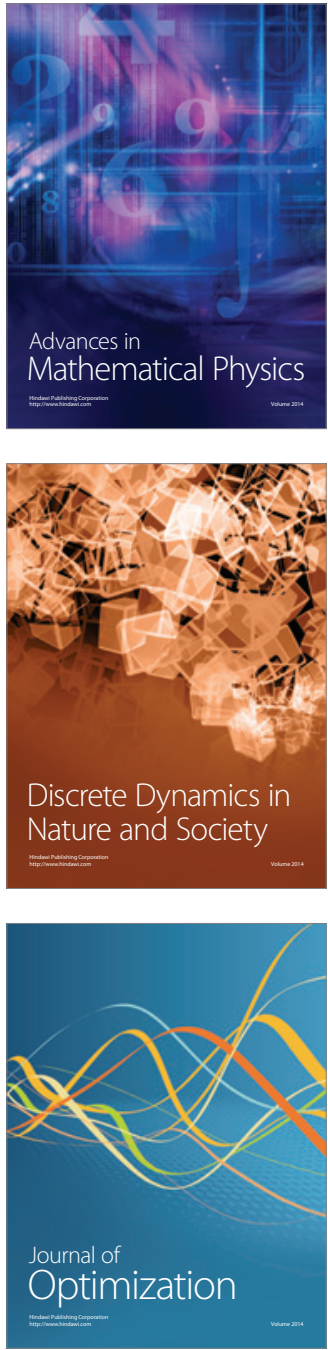